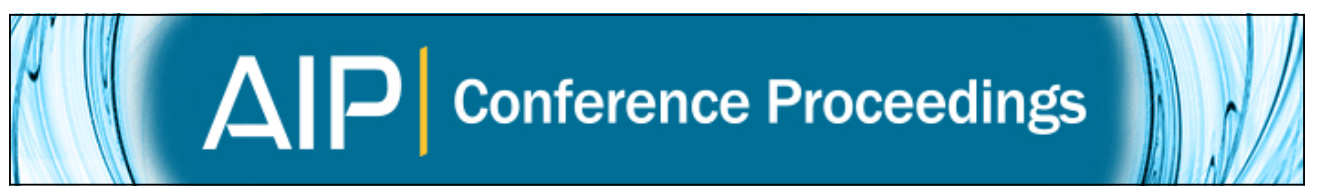

\title{
Low-Latency Detection of Gravitational Waves
}

Shaun Hooper, Linqing Wen, David Blair, Shin Kee Chung, Yanbei Chen, and Jing Luan

Citation: AIP Conference Proceedings 1246, 211 (2010); doi: 10.1063/1.3460207

View online: http://dx.doi.org/10.1063/1.3460207

View Table of Contents:

http://scitation.aip.org/content/aip/proceeding/aipcp/1246?ver=pdfcov

Published by the AIP Publishing

Articles you may be interested in

Electromagnetic to Gravitational wave Conversion via Nuclear Holonomy

AIP Conf. Proc. 1103, 524 (2009); 10.1063/1.3115561

Gravitational Waves in the Hyperspace

AIP Conf. Proc. 969, 1055 (2008); 10.1063/1.2844943

Dynamic Matched Filter for Gravitational Wave Detection

AIP Conf. Proc. 751, 236 (2005); 10.1063/1.1891562

Toward gravitational wave detection

AIP Conf. Proc. 523, 451 (2000); 10.1063/1.1291910

Answer to Question \#49. Why c for gravitational waves?

Am. J. Phys. 65, 590 (1997); 10.1119/1.18664 


\title{
Low-Latency Detection of Gravitational Waves
}

\author{
Shaun Hooper*, Linqing Wen*, , David Blair*, Shin Kee Chung*, \\ Yanbei Chen** and Jing Luan** \\ ${ }^{*}$ School of Physics, The University of Western Australia, \\ 35 Stirling Hwy, Crawley 6009 WA Australia \\ $\dagger$ International Centre for Radio Astronomy Research, The University of Western Australia \\ ${ }^{* *}$ Division of Physics, Mathematics and Astronomy, Caltech, Pasadena, CA 91125, USA
}

\begin{abstract}
Recent advances in ground-based interferometric gravitational wave detectors have produced a high level of confidence in detection of transient gravitational wave signals generated by coalescing neutron star or black hole binaries. The combination of gravitational wave data and associated electromagnetic radiation observed by telescopes will allow precision testing of general relativity, including measurement of the speed of gravitational waves. We present a new method of detection based on Infinite Impulse response (IIR) filtering with the goal of computing signal to noise ratios in near real time (low-latency). We then apply a set of IIR filters to the incoming detector data and coherent sum the outputs to produce near optimal signal to noise ratio. Our results show a computation time speed-up of $>50$ times compared with standard time-domain matched filter methods.
\end{abstract}

Keywords: General Relativity, Gravitational Waves, Data Analysis

PACS: 04.25.nx, 04.30.Db, 04.80.Cc, 04.80.Nn, 95.55.Ym, 95.85.Sz

\section{INTRODUCTION}

It is well known that Einstein's field equations allow for the propagation of disturbances through space-time. These disturbances are known as gravitational waves, and are generated whenever a massive quadrupole moment is accelerated. An example of the quadrupole moment is a compact binary system (including binary neutron stars, binary black holes and black hole-neutron star binaries). The gravitational waveform generated from a compact binary coalescence (CBC) event has an amplitude and phase function $\phi(t)$ that is directly proportional to the orbital phase of the binary. The rate of coalescence, which is primary determined by the masses of the bodies, can be very well approximated by the post-Newtonian approximation ([1, and references therein]).

The sensitivity of current interferometric gravitational wave detectors is now at a level where the detection of gravitational waves from CBC events is possible. A major upgrade of the current initial LIGO detectors to advanced LIGO is scheduled to happen after the current science run (end of 2010), and is due to go online in 2015. This upgrade will result in a sensitivity factor improvement of 10 relative to initial LIGO [2]. Hence, advanced LIGO will be able to detect GW sources within a volume of space one thousand times larger than that of initial LIGO (out to $\sim 200-300 \mathrm{Mpc}[3]$ ). The sensitivity will also reach a lower frequency of $10 \mathrm{~Hz}$, compared to initial LIGO's $40 \mathrm{~Hz}$.

It is well known that the use of a matched filter is the optimal method of detecting known waveforms in noisy detector data [4]. The matched filter output $z$ is a correlation

CP1246, Frontiers of Fundamental and Computational Physics - Proceedings of the $10^{\text {th }}$ International Symposium edited by J. G. Hartnett and P. C. Abbott

(C) 2010 American Institute of Physics 978-0-7354-0793-0/10/\$30.00 
of a waveform template $h(t)$ (that terminates at $t=0$ as it is a transient signal) and the detector data $s(t)$ weighted by the an appropriate whitening function $S_{n}$.

$$
z(t)=2 \int_{-\infty}^{t} x\left(t^{\prime}\right) h^{*}\left(t^{\prime}-t\right) d t^{\prime}=2 \int_{-\infty}^{\infty} \frac{\tilde{s}(f) \tilde{h}^{*}(f)}{S_{n}(|f|)} e^{2 \pi i f t} d f
$$

Here $S_{n}(f)$ is the one-sided noise spectral density and $x(t)=\mathscr{F}^{-1}\left(\tilde{s}(f) / S_{n}(f)\right)$ is the whitened detector data. In the second equality, we have moved from the time domain to the frequency domain via the cross-correlation theorem. Here the tilde represents the Fourier transform. Although it is more computationally efficient to calculate the correlation $z$ in the frequency domain rather than the time domain, there is a latency involved as one needs to wait until there is enough detector data to apply a Fourier transform (the current pipeline uses data segments of 2048 seconds [5]).

The signal to noise ratio (SNR) is proportional to $z$ and a high value indicates that a predicted waveform is likely to be present in the data.

\section{TIME DOMAIN FILTERING}

Here we present an alternative method to calculate an approximate SNR ratio using a time domain method, with the goal of low-latency. Consider first the discretisation of the equation (1)

$$
z_{k}=\sum_{j=-\infty}^{k} x_{j} h_{j-k}
$$

Of course in practise $h$ is of length $N$ rather that infinite. Each $z_{k}$ can then be computed by $N$ calculations. In this case, eq. 2 is also known as a Finite Impulse Response (FIR) filter. We introduce an alternative digital filter, the Infinite Impulse Response (IIR) filter,

$$
y_{k}=a_{1} y_{k-1}+b_{0} x_{k}
$$

where $y_{k}$ is the filter output at time step $k, x_{k}$ is the filter input, and $a_{1}$ and $b_{0}$ are complex coefficients. If $b_{0} a_{1}^{n}=h_{n}$, then $y_{k}$ is equal to $z_{k}$. Finding complex coefficients $b_{0}, a_{1}$ that correspond to $h$ can be done by approximating $h$ by a sum of $M$ exponentially increasing, constant frequency sinusoids,

$$
h_{\text {approx }}(t)=\Re \sum_{l}^{M} b_{0, l} a_{1, l}^{t_{l}-t} \Theta\left(t_{l}-t\right)
$$

Each sinusoid is created by performing a Taylor expansion of the phase function $\phi(t)$ to linear order about a time $t_{l}$. The sinusoid will have a frequency corresponding to the frequency of $h(t)$ that occurs at time $t_{l}$. The next sinusoid is chosen by finding $t_{l+1}=t_{l}+\Delta t_{l}$ where $\left|\frac{1}{2} \ddot{\phi}\left(t_{l}\right) \Delta t_{l}^{2}\right|=\varepsilon$, and $\varepsilon$ is a value chosen to be sufficiently small to reduce second-order effects. Each sinusoid needs to be multiplied by a damping/window function $e^{\gamma_{l}\left(t-t_{l}\right)} \Theta\left(t_{l}-t\right)$ that will suppress the interference of previous sinusoids. Here we chose $\gamma_{l}=\eta / \Delta t_{l}$. Fig. 1 shows how the summation of these sinusoids evaluated at time $t_{l}$ can approximate the original waveforms. 


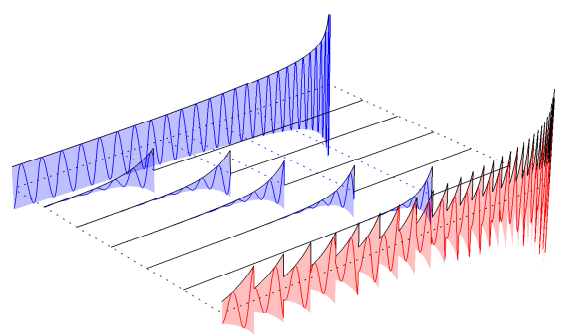

FIGURE 1. The back row (blue) is the exact waveform $h(t)$. The exponentially increasing sinusoids (middle blue's) are the Taylor expansion of the waveform about time $t_{l}$ to first order, and multiplied by an exponential window function. The linear addition of the sinusoids can then approximate the waveform, shown in the front row (red).

The whitened detector data $x_{k}$ filtered by each IIR filters (see eq. 3 ) using coefficients $a_{1, l}, b_{0, l}$ produces $M$ filter outputs $y_{k, l}$ 's. A sum of the all the filter outputs, each shifted forward by time $t_{l}$, then produces $z_{k}$, which is exactly equivalent to the correlation of $h_{\text {approx }}(t)$ and whitened detector data $x$.

\section{RESULTS}

We test the ability of our IIR method to recover a SNR equivalent to that calculated using the optimal matched filtering method by filtering mock whitened detector data. The mock whitened detector data is generated by embedding a signal $s(t)$ with the waveform $h(t)$, and then whitening it to produce the filter input $x(t)$. We define the SNR overlap as the ratio of the SNR produced by IIR filtering $x(t)$, and the SNR obtained from matched filtering.

We first imbed the signal with a waveform that would be created by $1.4-1.4 M_{\odot}$ binary. We choose to generate IIR filters starting at a time $t_{l}$ when the waveform has frequency of $40 \mathrm{~Hz}$ (the lower limit of initial LIGO's sensitivity bandwidth). For our chosen value of $\varepsilon, 200$ IIR filters cover this frequency range. Applying the IIR filters to the mock signal and coherently adding the filter outputs recovers a maximum SNR overlap of $99 \%$.

The number of IIR filters is wholly dependant on the value of $\varepsilon$. Generally speaking, a greater number of filters corresponds to greater frequency response range, which means a higher SNR overlap. However the choice of the $\eta$ will alter the SNR overlap due to unwanted contributions from other filters. We show the maximum SNR overlap as a function of the number of filters in figure 2. Also shown is the maximum SNR overlap for other mass pairs, for a given $\eta$.

In order to show the computational speed-up of from calculating the SNR in the time domain using the IIR method in comparison with the FIR method (equation (2)), we timed both methods and calculated the speed-up factor (the ratio of FIR and IIR calculation times). The speed-up demonstrates a real advantage when lower starting frequencies are used. A lower starting frequencies correspond to a longer signal. This means the calculation time of an FIR will be longer, but only a few extra IIR filters are required. We show this speed-up factor in Fig. 3. 


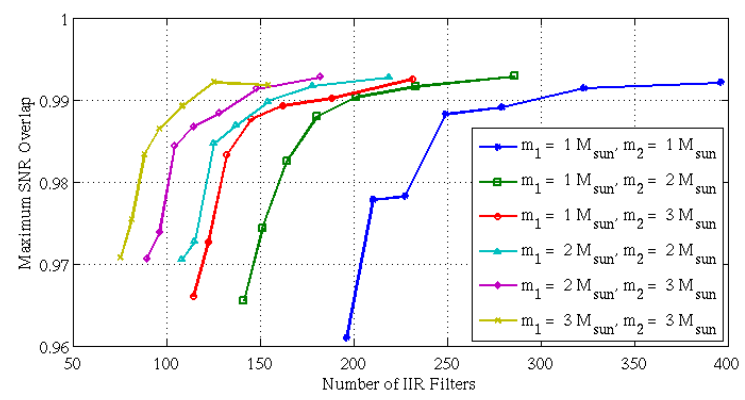

FIGURE 2. Here we show the number of filters per waveform (horizontal axes) required to calculate a SNR comparable to the optimal matched filter method (maximum SNR overlap, plotted on the vertical axis). We show the results for 5 different mass pairs, ranging from $1-3 M_{\odot}$.

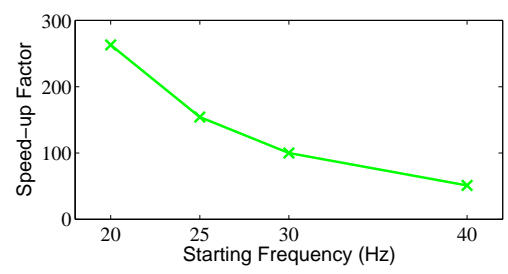

FIGURE 3. The speed-up factor (FIR calculation time vs IIR calculation time) is shown as a function of starting frequency. Here, starting frequency directly links to the length of the signal. Here we see that there is a significant advantage of using the IIR method over the FIR at lower starting frequencies.

\section{CONCLUSION}

In this paper we have demonstrated the use of infinite impulse response (IIR) filtering to calculate the SNR of whitened gravitational wave detector data. Our results are comparable to the optimal matched filtering method ( $>99 \%)$, but can be calculated in the time domain, with low-latency (near real time). In terms of processing speed, we have empirically demonstrated that the IIR filters offer a improvement over the FIR filter, especially when searching for waveforms with lower frequencies. Hence the IIR method will be particularly advantageous for the detection of gravitational waves using advanced LIGO, which is will be able to access frequencies down to $10 \mathrm{~Hz}$.

\section{REFERENCES}

1. S. Fairhurst, and P. Brady, Class. Quant. Grav. 25, 105002 (2008)

2. J. R. Smith, and for the LIGO Scientific Collaboration, Class. Quant. Grav. 26, 114013-+ (2009) .

3. Advanced LIGO Reference Design, LIGO Tech. Rep. M060056 (2007), URL http: / / www . I igo . caltech.edu/docs/M/M060056-08/M060056-08.pdf.

4. L. A. Wainstein, and V. D. Zubakov, Extraction of Signals from Noise, Prentice-Hall, 1962.

5. B. Abbott, et al., Phys. Rev. D73, 062001 (2006) 